\section{O censo escolar como estratégia de busca de crianças e adolescentes em estudos epidemiológicos}

\author{
The school census as a search strategy for children \\ and adolescents in epidemiological studies
}

\author{
${ }^{1}$ Universidade Federal de \\ Mato Grosso, Cuiabá, Brasil. \\ 2 Universidade do Estado do \\ Rio de Janeiro, Rio de Janeiro, \\ Brasil. \\ 3 Universidade Federal do \\ Rio de Janeiro, Rio de Janeiro, \\ Brasil. \\ Correspondência \\ R. M. V. Gonçalves-Silva \\ Universidade Federal de Mato \\ Grosso. \\ Av. Fernando Corrêa da Costa \\ 2367, Cuiabá, MT 78060-900, \\ Brasil. \\ reveras@uol.com.br
}

\section{Abstract}

Low-cost alternatives that allow for the monitoring of population groups are important strategies in conducting cohort studies. This paper presents the procedures for the use of the National School Census as a search tool for children and adolescents in follow-up studies. The study was conducted among adolescent students who took vaccinations in clinics in Cuiabá, Mato Grosso State, Brazil in 1999. A total of $86.8 \%$ were identified in the census and $0.2 \%$ in the Mortality Information System. Of those identified, 1,716 were interviewed, representing a follow-up rate of $71.4 \%$. In this study, the school census proved to be an effective method for finding students.

Adolescent; Longitudinal Study; Information System

\author{
Regina Maria Veras Gonçalves-Silva ${ }^{1}$ \\ Rosely Sichieri 2 \\ Márcia Gonçalves Ferreira 1 \\ Rosângela Alves Pereira 3 \\ Ana Paula Muraro 1 \\ Naiara Ferraz Moreira 1 \\ Joaquim Gonçalves Valente 2
}

\section{Introdução}

Estudos de coorte são fundamentais para o entendimento das consequências, em longo prazo, dos eventos que incidem em fases precoces da vida. Entretanto, este desenho de estudo é pouco conduzido em países em desenvolvimento, devido, provavelmente, aos custos elevados e à dificuldade de seguir os participantes da pesquisa.

No Brasil, as coortes de nascimento de Pelotas no Rio Grande do Sul (1982, 1993, 2004) 1, que representam o maior estudo de acompanhamento já realizado no país, têm gerado inúmeras publicações disponíveis em bases de dados de grande repercussão na literatura científica mundial 2 .

A construção de novas coortes no Brasil é de fundamental importância, dada a particularidade das condições de vida da população, frente à transição epidemiológica e nutricional que se apresenta, pois se entende que o custo social do desenvolvimento tem implicações importantes nesses processos. Sabe-se que a relação entre exposição e desfecho está fortemente relacionada às características populacionais e aos fatores ambientais, particularmente, às condições socioeconômicas. Além disso, informações obtidas em estudos nacionais são mais eficientes para a tomada de decisões em relação a medidas de controle e prevenção de enfermidades.

Desta forma, a busca de alternativas viáveis e de menor custo para tornar possível o acom- 
panhamento de um grupo populacional pode significar uma estratégia interessante para a condução de estudos de coorte e a utilização do censo escolar pode ser uma ferramenta útil para a realização de estudos longitudinais com crianças e adolescentes. Assim, este trabalho apresenta os procedimentos aplicados na busca e localização dos adolescentes, além de informar os resultados desta busca e as perdas de seguimento, apresentando as estratégias aplicadas para minimizar a não-resposta.

\section{Métodos}

Entre os meses de maio de 1999 e janeiro de 2000, foi realizado um estudo com crianças menores de cinco anos, que frequentaram os postos de saúde do Município de Cuiabá, Mato Grosso para vacinação, com o objetivo de estudar possíveis associações entre o tabagismo passivo e o estado nutricional ${ }^{3}$. Dez anos após essa primeira avaliação, as crianças, agora com idade entre 10 e 15 anos, foram localizadas para realização de novos estudos.

No estudo de base foram avaliadas 2.405 crianças. Para localização da criança no censo escolar foram utilizadas as variáveis levantadas no estudo inicial: data de nascimento, bairro, cidade e estado onde nasceu, endereço do domicílio, nome completo dos pais ou responsáveis e seus respectivos telefones, fixo e/ou celular.

\section{Estratégias de busca dos adolescentes}

Em 2009, iniciou-se estudo para avaliar a viabilidade do seguimento dessa coorte em Cuiabá, que é uma cidade em crescimento e com baixa taxa de emigração, o que favoreceria a localização das crianças.

Considerando que as crianças estavam em idade escolar (10 a 15 anos), elegeu-se a escola como o local apropriado para fazer o seguimento das crianças, pelo fato de que a cobertura escolar para aquela faixa etária em Cuiabá era de 97,3\%.5 Para a realização da busca, a opção foi utilizar o censo escolar, EducaCenso, que é um levantamento de dados estatístico-educacionais, de âmbito nacional, realizado todos os anos sob a coordenação do Instituto Nacional de Estudos e Pesquisas Educacionais Anísio Teixeira (INEP), com o apoio das Secretarias Estaduais de Educação (http://www.inep.gov.br/básica/censo/ default.asp, acessado em 14/Jul/2010).

O projeto de pesquisa, aprovado pelo Comitê de Ética do Hospital Universitário Júlio Müller da Universidade Federal de Mato Grosso (HUJM/ UFMT), foi enviado para a Secretaria de Esta- do de Educação de Mato Grosso, que permitiu a realização da busca eletrônica dos adolescentes pelo programa de banco de dados do INEP, que foi realizada por técnicos responsáveis pelo censo escolar. O censo permite identificar as escolas públicas e privadas de todo o país e seus respectivos endereços, onde o adolescente está matriculado.

O primeiro contato com as escolas foi feito com os diretores, que eram informados sobre os objetivos da pesquisa, sendo solicitada a sua autorização para o contato com os adolescentes que seriam entrevistados. A receptividade por parte dos diretores, tanto das escolas públicas, quanto das privadas foi muito boa, não havendo nenhum caso de não cooperação.

A partir da permissão do diretor, o entrevistador abordava o adolescente esclarecendo-o sobre a pesquisa e solicitando que encaminhasse aos pais o termo de consentimento, para ser assinado pelo responsável. Somente após a entrega do termo de consentimento, devidamente assinado, a entrevista era realizada. Para a devolução do termo de consentimento, pelo adolescente, foram realizadas, em média, até três visitas às escolas. Para minimizar a não resposta foram realizadas as seguintes estratégias: revisitas as escolas; contato telefônico com os pais, a partir de informações fornecidas pelas escolas; atualização constante da busca no banco de dados do censo escolar e envio de carta de apresentação aos pais, anexo ao termo de consentimento, para esclarecimento sobre a pesquisa.

Outra fonte de busca dos adolescentes não localizados por meio do censo escolar foi a procura no Sistema de Informações sobre Mortalidade (SIM) do Ministério da Saúde. A busca foi autorizada, pelo superintendente da Vigilância em Saúde da Secretaria de Estado da Saúde e realizada por um técnico deste setor, após entrega dos documentos solicitados, que incluiu o parecer do Comitê de Ética. Nesse sistema, os dados são organizados por Unidades da Federação e estão disponíveis anualmente e podem ser acessados na página de Internet do Departamento de Informática do SUS (DATASUS). Para localizar o óbito são necessárias informações sobre o município de ocorrência e local de residência. A busca no SIM foi realizada a partir do ano de 1999 com base nas informações da Secretaria de Estado de Saúde, que forneceu a relação dos óbitos que ocorreram em Mato Grosso desde então. Somente, a partir de 2006 foram informados para o sistema, os óbitos de residentes do estado ocorridos em outras unidades da federação. 
Os participantes da pesquisa foram devidamente esclarecidos sobre os propósitos do estudo e os pais ou responsáveis assinaram o Termo de Consentimento Livre e Esclarecido, conforme preconiza a Resolução $n^{\circ}$. 196/96 do Conselho Nacional de Saúde. Essa pesquisa não incluiu nenhum procedimento invasivo que oferecesse riscos aos participantes. O projeto de pesquisa foi aprovado pelo Comitê de Ética do Hospital Universitário Júlio Muller, da Universidade Federal de Mato Grosso, sob o protocolo n. 651/CEP/HUJM/09.

\section{Resultados}

Das 2.405 crianças investigadas no estudo de base, $86,8 \%$ (2.088) foram localizadas por meio do censo escolar, sendo 72,4\% (1.742) localizados em Cuiabá e Várzea Grande (cidade integrada geográfica e economicamente a Cuiabá), 7,6\% em cidades do interior do Estado de Mato Grosso e $6,8 \%$ em outras cidades do país. Cerca de $13 \%$ dos adolescentes não foram localizados pelo censo escolar. A busca no SIM permitiu a identificação de cinco óbitos (Figura 1). No total, foram localizados no censo escolar e no SIM, 2093 adolescentes (87\%).

Dentre os adolescentes localizados, foram entrevistados 1.716, representando uma taxa de seguimento de $71,4 \%$. Foram entrevistados em Cuiabá e Várzea Grande 92,1\% (1.605). Em 17 cidades próximas à capital foram entrevistados 74 dos 123 adolescentes localizados (60\%). Nas outras cinco capitais visitadas - Brasília (Distrito Federal), Goiânia (Goiás), Rio de Janeiro, São Paulo e Campo Grande (Mato Grosso do Sul) - foram avaliados 37 adolescentes dos 56 localizados (66\%). A dispersão dos adolescentes em outras cidades do país $(6,9 \%)$ impossibilitou a entrevista, por questões de logística (Figura 1).

\section{Discussão}

A busca de estudantes utilizando o censo escolar mostrou que é possível a localização dos mesmos para estudos de seguimento. Essa é uma ferramenta inovadora de busca de estudantes para analisar eventos relacionados à saúde, que pelo que se tem conhecimento não foi utilizado, no Brasil, até a presente data.

A estratégia de localização dos adolescentes pelo censo escolar pode trazer inúmeras contribuições para o estudo de eventos ocorridos precocemente, pois permite seu acompanhamento até o final da vida escolar. Considerando que até os 17 anos de idade, 80,3\% dos jovens estão frequentando as escolas na Região Centro-oeste, existe, neste caso, uma possibilidade de seguimento que poderá incrementar a elaboração de novos projetos com crianças e adolescentes (http://mteseusmunicipios.com.br/NG/conte údo.php?sid=145\&Cid=1484, acessado em 14/ Jul/2010).

No entanto, a espera pelo término do cadastramento dos alunos, que se dá apenas no segundo semestre de cada ano é um fator limitante. Outra dificuldade para a localização do estudante são os erros de digitação do nome e data de nascimento da criança, quando da inserção de dados no sistema realizados pelas escolas.

Considerando-se a elevada cobertura do SIM na Região Centro-oeste $(84,1 \%) 4$, pode-se considerar consistentes os dados de mortalidade levantados no presente estudo. No entanto, até 2005, o sistema não provia dados sobre os óbitos de residentes de Mato Grosso ocorridos em outros estados, o que pode ter resultado em alguma perda de informação.

A integração dos sistemas disponíveis na área da saúde representa um recurso importante para o desenvolvimento de estudos de seguimento de base populacional. Nesse sentido, a inserção do EducaCenso como uma base de dados integradora do conjunto de sistemas de informação, poderia se constituir em importante contribuição para o desenvolvimento de estudos epidemiológicos. Para tanto é fundamental garantir que o nome da criança, a data de nascimento e o nome da mãe sejam muito bem coletados na linha de base. 
Figura 1

Representação esquemática da busca e avaliação dos adolescentes da coorte.

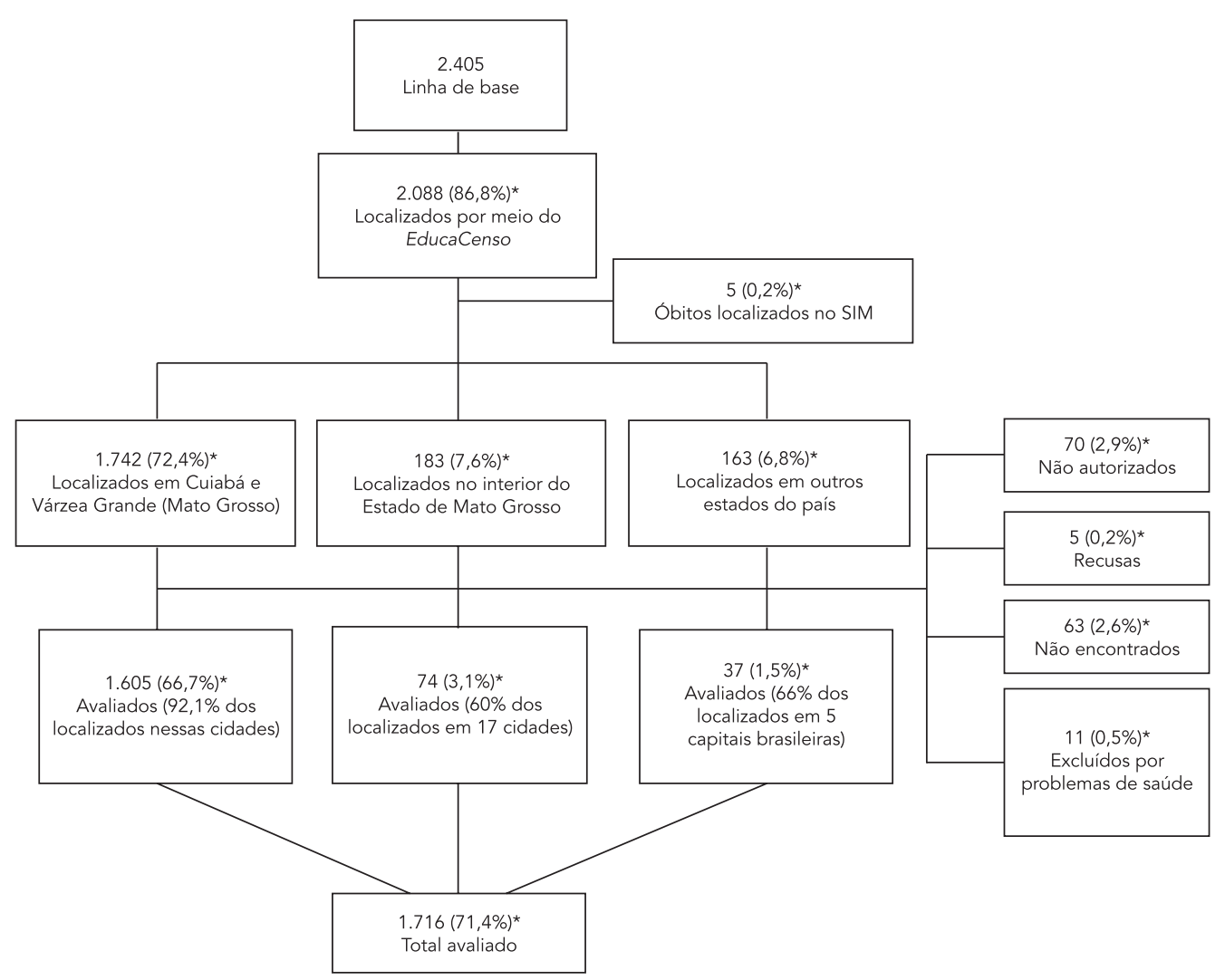

* Percentual relativo à linha de base.

\section{Resumo}

Alternativas de baixo custo que possibilitem o acompanhamento de grupos populacionais constituem estratégias importantes na condução de estudos de coorte. Este trabalho apresenta os procedimentos para o uso do censo escolar como instrumento de busca de crianças e adolescentes em estudos de seguimento. O estudo foi realizado entre os estudantes adolescentes que frequentaram os postos de saúde de Cuiabá, Estado de Mato Grosso, Brasil, para vacinação em 1999.
Foram localizados $86,8 \%$ de adolescentes com o uso do censo escolar e 0,2\% pelo Sistema de Informações sobre Mortalidade. Dentre os adolescentes localizados, foram entrevistados 1.716, representando uma taxa de seguimento de 71,4\%. Neste estudo, o censo escolar mostrou ser um método efetivo na busca de estudantes.

Adolescente; Estudos Longitudinais; Sistemas de Informação 


\section{Colaboradores}

R. M. V. Gonçalves-Silva participou de todas as etapas da pesquisa e escreveu o artigo. R. Sichieri participou da concepção, do desenho e da revisão do artigo. M. G. Ferreira participou da coleta, da interpretação dos dados e da revisão do artigo. R. A. Pereira participou da concepção e da revisão do artigo. A. P. Muraro participou da busca dos adolescentes no EducaCenso, da coleta, digitação e interpretação dos dados. N. F. Moreira participou da busca dos adolescentes no EducaCenso, da coleta, digitação e interpretação dos dados. J. G. Valente participou da concepção e da revisão do artigo.

\section{Referências}

1. Barros AJD, Santos IS, Matijasevich A, Araújo CL Gigante DP, Menezes AMB, et al. Methods used in the 1982, 1993, and 2004 birth cohort studies from Pelotas, Rio Grande do Sul State, Brazil, and a description of the socioeconomic conditions of participants' families. Cad Saúde Pública 2008; 24 Suppl 3:S371-80.

2. Sichieri R, Benício MHD, Barreto SM, Lima-Costa MF. Editorial. Rev Saúde Pública 2008; 42 Suppl 2:1-2.

3. Gonçalves-Silva RMV, Valente JG, Lemos-Santos MGF, Sichieri R Tabagismo no domicílio e baixa estatura em menores de cinco anos. Cad Saúde Pública 2005; 21:1540-9.

\section{Agradecimentos}

À Secretaria de Estado de Educação de Mato Grosso, na pessoa da Profa. Josinete Aparecida da Silva Ferraz, Coordenadora do Censo Escolar em Mato Grosso, por ter permitido a localização dos estudantes no EducaCenso. Ao CNPq (processo no ${ }^{\circ}$ 471063/2009-6) e à Fapemat (processo $\mathrm{n}^{\circ}$. 446298/2009), pelo apoio financeiro ao projeto.
4. Coordenação de Acompanhamento e Avaliação de Atenção Básica, Departamento de Atenção Básica, Secretaria de Atenção à Saúde, Ministério da Saúde. Integração de informações do SIAB, SIM e SINASC nos sistemas locais de saúde. Brasília: Ministério da Saúde; 2004.

Recebido em 03/Jun/2011

Versão final reapresentada em 28/Out/2011 Aprovado em 23/Nov/2011 